\title{
Inteligencia emocional y ajuste psicosocial en la adolescencia: El papel de la percepción emocional
}

\author{
José M. Salguero ${ }^{1}$, Pablo Fernández-Berrocal ${ }^{1}$, Desireé Ruiz-Aranda ${ }^{1}$, Ruth \\ Castillo $^{1}$ y Raquel Palomera ${ }^{2}$ \\ ${ }^{1}$ Universidad de Málaga, ${ }^{2}$ Universidad de Cantabria (España)
}

La inteligencia emocional (IE), definida como la habilidad para percibir, usar, comprender y regular las emociones, se ha mostrado como una variable importante a la hora de explicar el ajuste psicosocial de las personas. Pese a ello, la mayor parte de la investigación en torno a la IE ha sido realizada con muestras de población adulta y son escasos los trabajos que, utilizando medidas de ejecución, exploren su papel en la adolescencia. El objetivo del presente estudio fue el de analizar la influencia de la percepción emocional, una de las habilidades recogidas dentro de la IE, sobre el ajuste personal y social de una muestra de adolescentes españoles. Un total de 255 alumnos completaron una tarea de ejecución diseñada para evaluar la percepción emocional en la adolescencia así como diferentes medidas de ajuste social y personal. Los resultados de los análisis de correlación y regresión mostraron cómo los adolescentes con una mayor habilidad para reconocer los estados emocionales de los demás informaron de mejores relaciones sociales con iguales y padres, menor tensión en sus relaciones sociales, así como de un mayor nivel de confianza y competencia percibida. Además, estos resultados se mantuvieron tras controlar los efectos de la edad, el sexo y la personalidad. Se discuten los resultados en el contexto de la investigación sobre la inteligencia emocional y se proponen implicaciones prácticas para el diseño de programas de prevención y tratamiento.

Palabras clave: Inteligencia emocional, percepción emocional, relaciones sociales.

Emotional intelligence and psychological and social adjustment in adolescence: The role of emotional perception. Emotional intelligence (IE), defined as the ability to perceive, use, understand and regulate emotions, has shown a positive influence on the people's psychological and social adjustment in the specialized literature. However, most of the work has been carried out with adult samples and few investigations have examined this issue with adolescent population. The aim of this paper was to analyze the influence of emotional perception, an ability of EI, on the adolescent's personal and social adjustment. A sample of 255 Spanish adolescents completed a maximum performance test of emotional perception and different scales measuring personal and social adjustment. Results of correlation and regression analyses showed that adolescents with higher ability to perceive emotions reported having better relationships with peers and parents, lower social stress, higher self-confidence and higher perceived competence. Moreover, emotional perception significantly predicted adolescents' adjustment even when the effects of sex, age, and personality were controlled for. The findings suggest that emotional intelligence is a promising predictor of adolescent adjustment and may serve as a useful resource for preventive and treatment interventions.

Key words: Emotional intelligence, emotional perception, social relationships, well-being.

Correspondencia: José M. Salguero Noguera. Facultad de Psicología. Campus de Teatinos s/n. 29071. Málaga. Email: jmsalguero@uma.es 
Las emociones poseen un papel fundamental en nuestra supervivencia. No sólo están implicadas en la activación y coordinación de los cambios fisiológicos, cognitivos y conductuales necesarios para ofrecer una respuesta efectiva a las demandas del ambiente (Cosmides y Tobby, 2000; Nesse, 1990), sino que son elementos fundamentales en la toma de decisiones (Damasio, 1994; Katelaar y Todd, 2001; Overskeid, 2000) y una fuente útil de información acerca de la relación entre el individuo y su medio (Fridja, 1988; Mayer y Salovey, 1997; Plutchik, 1984; Salovey, Mayer, Goldman, Turvey y Palfai, 1995).

Siguiendo esta concepción evolucionista y funcionalista de las emociones, la literatura científica ha prestado especial interés en los últimos años al estudio de las diferencias individuales en la habilidad para procesar y utilizar la información emocional partiendo de la hipótesis de que aquellas personas capaces de percibir y comprender las emociones, asignar un significado a la experiencia emocional y regular sus sentimientos tendrán una mejor adaptación psicológica y social (Ciarrochi, Chan, Caputi y Roberts, 2001; Martorell, González, Rasal y Estellés, 2009; Ramos, Fernández-Berrocal, y Extremera, 2007). Estas habilidades han sido conceptualizadas de forma general bajo el término Inteligencia Emocional (IE) (Mayer y Salovey, 1997; Salovey y Mayer, 1990). Aunque existen diferentes aproximaciones teóricas a la IE (para una revisión ver Mayer, Salovey y Caruso, 2000), su definición más extendida pertenece al modelo de habilidad de Mayer y Salovey (1997).

El modelo de habilidad define la IE como "la habilidad para percibir, valorar y expresar las emociones con exactitud; la habilidad para acceder y generar sentimientos que faciliten el pensamiento; la habilidad para entender la emoción y el conocimiento emocional; y la habilidad para regular las emociones y promover el crecimiento emocional e intelectual" (Mayer y Salovey, 1997, p.10). Se trata por tanto de cuatro conjuntos de habilidades diferentes -percepción emocional, facilitación emocional, comprensión emocional y regulación emocional- las cuales se establecen de forma jerárquica, esto es, la primera de las habilidades (percepción emocional) supone el nivel más básico de la jerarquía, de menor complejidad aunque necesaria para conseguir las habilidades superiores, mientras que la habilidad de mayor nivel (regulación emocional) será la de mayor complejidad, aunque para llegar a ella necesitamos un cierto dominio de las habilidades anteriores.

Una de las principales ventajas del modelo de habilidad de la IE ha sido la posibilidad de evaluar esta inteligencia utilizando instrumentos construidos con diferentes procedimientos (Extremera, Salguero, Fernández-Berrocal y Ruiz-Aranda, 2009). De ellos, destacan los test de ejecución. Este tipo de pruebas se centran en medir las destrezas emocionales incluidas en la IE evaluando, más que la percepción que cada persona posee sobre su habilidad, el nivel de ejecución actual que muestra en diferentes tareas emocionales. El test de ejecución más utilizado en la investigación de la IE desde 
el modelo de habilidad es el MSCEIT (Mayer-Salovey-Caruso Emotional Intelligence Test, Mayer, Salovey y Caruso, 2001).

Los estudios llevados a cabo con el MSCEIT han mostrado la existencia de relaciones significativas entre la IE y el ajuste personal y social (Mayer, Roberts y Barsade, 2008). En lo que respecta al comportamiento social, una mayor IE se ha relacionado con una mejor percepción de competencia social y un menor uso de estrategias interpersonales negativas (Brackett, Rivers, Shiffman, Lerner y Salovey, 2006; Lopes et al., 2004), una mayor calidad de las relaciones sociales (Extremera y Fernández-Berrocal, 2004), o mejores relaciones familiares y de pareja (Brackett et al., 2005); además, las personas con una mayor IE son percibidas por los demás como más agradables, empáticas y sociables (Lopes, Salovey, Cote y Beers, 2005). En lo que respecta al ajuste personal, la IE se ha asociado de forma significativa con variables criterio tan importantes como el nivel de autoestima (Brackett et al., 2006), el bienestar (Brackett y Mayer, 2003) o una menor sintomatología depresiva (Extremera, FernándezBerrocal, Ruiz-Aranda y Cabello, 2006). Estos datos aportan evidencias de la implicación que un adecuado manejo de las emociones supone para nuestro desarrollo psicosocial. Las personas emocionalmente inteligentes no sólo poseen una mayor capacidad para percibir, comprender y regular sus emociones, repercutiendo de forma positiva en su bienestar personal, sino que también son capaces de generalizar estas habilidades a las emociones de los demás favoreciendo de este modo sus relaciones sociales, familiares e íntimas.

A pesar de la importancia de estos resultados, la mayor parte de la investigación con el MSCEIT ha sido realizada con muestras de población adulta y son escasos los trabajos que analizan la implicación de la IE en la adolescencia utilizando medidas de ejecución. Conocer la influencia de las habilidades emocionales en etapas más tempranas del desarrollo puede ser un objetivo de gran importancia por diferentes motivos: primero, porque permitiría analizar la forma en que dichas habilidades evolucionan a lo largo del ciclo vital; segundo, porque nos ayudaría a explicar las diferencias en el nivel de adaptación de los adolescentes; tercero, porque, de corroborarse la implicación de la IE en el ajuste psicológico y social de niños y adolescentes, el entrenamiento de estas habilidades supondría una prometedora vía para la prevención y el tratamiento de problemas emocionales y/o sociales.

En este contexto, el objetivo del presente trabajo fue explorar la influencia que la percepción emocional, la rama más básica del modelo de IE de Mayer y Salovey (1997), tiene sobre el funcionamiento psicosocial de una muestra de adolescentes españoles. Para ello, evaluamos el nivel de percepción emocional interpersonal utilizando una tarea de ejecución diseñada específicamente para población adolescente.

Las emociones nos aportan información relevante acerca de los pensamientos e intenciones de otras personas y nos permiten dirigir de forma efectiva nuestros 
encuentros sociales (Keltner y Haidt, 2001; Lopes et al., 2004). En este sentido, la habilidad para detectar y comprender las señales emocionales ajenas se presenta como un requisito importante a la hora de determinar un comportamiento social competente (Izard, 2001; Salovey y Mayer, 1990). Aunque la capacidad para "leer" emociones en los rostros de otras personas es uno de los atributos que compartimos los humanos a través de diversas culturas (Ekman, 2003), existen diferencias importantes en el grado en las personas son capaces de poner en marcha esta habilidad. En la presente investigación trabajamos bajo la hipótesis de que aquellos adolescentes con una mayor habilidad para percibir emociones en los demás mostrarán un mejor ajuste personal y social. Puesto que diferentes autores han reclamado la necesidad de controlar el efecto de dimensiones de personalidad que comparten dominios emocionales y que han demostrado de forma consistente su relación con variables relacionadas con el bienestar personal y social (tales como la extraversión y el neuroticismo) (Zeidner, Matthews y Roberts, 2008), analizaremos la influencia de la percepción emocional sobre las variables de ajuste psicosocial una vez controlado el efecto de estas dimensiones de personalidad.

\section{METODO}

\section{Participantes}

En este estudio participaron un total de 255 alumnos (49.4\% varones, 50.6\% mujeres) pertenecientes a diferentes centros de Enseñanza Secundaria Obligatoria de la provincia de Málaga. La media de edad fue de 13.43 años (desviación típica $=0.67$ ) con un rango de 12 a 15 años.

\section{Instrumentos}

Percepción emocional. Se evaluó la habilidad de percepción emocional mediante una tarea de ejecución diseñada para adolescentes y basada en el modelo de Inteligencia Emocional de Mayer y Salovey (1997). La tarea consiste en identificar el grado en que diferentes sentimientos son expresados por una persona a través de su expresión facial. Se utilizaron para ello 6 fotografías de los rostros de 6 adolescentes diferentes, en base a las cuáles cada persona debía indicar el grado de intensidad con que se expresaban los sentimientos de sorpresa, ira, tristeza, miedo, felicidad y asco (se utilizó una escala Lickert de 5 puntos, $1=$ "el sentimiento no está nada expresado en el rostro", 5= "el sentimiento es muy fuerte en el rostro"); la prueba consta, por tanto, de un total de 36 ítems. Siguiendo un procedimiento similar al utilizado para la corrección de otras medidas de ejecución de la IE (e.g. MSCEIT), obtuvimos un criterio experto en base a las respuestas aportadas por 16 psicólogos clínicos expertos en emociones, criterio que fue usado para determinar las respuestas correctas. La prueba de percepción emocional mostró adecuadas propiedades psicométricas, así, en una muestra 1.656 
adolescentes (43.3\% varones) con edades comprendidas entre los 12 y los 17 años $($ Media $=14.43$; Desviación típica $=1.56)$, obtuvo un alpha de Cronbach de .86 .

Ajuste psicosocial. Para evaluar el nivel ajuste personal y social de los participantes utilizamos el Sistema de evaluación de la conducta en niños y adolescentes (BASC; Reynolds y Kamphaus, 2004), en su versión de auto-informe para adolescentes. El BASC es una de las pruebas más reconocidas internacionalmente para la evaluación socio-emocional de niñas/os y adolescentes, tanto en el campo escolar cómo en el clínico. En este estudio, utilizamos tres subescalas referidas al funcionamiento social, en concreto: relaciones interpersonales (percepción de tener buenas relaciones sociales y amistades con las compañeras/os), relación con los padres (consideración positiva hacia las madres/padres y sentimientos de que ellos le estiman), y estrés social (sentimientos de estrés y tensión en las relaciones personales; sentimiento de ser excluido de las actividades sociales); y dos subescalas que evalúan el ajuste personal: sentimientos de incapacidad (percepción de no tener éxito en el colegio, dificultad para conseguir los propios objetivos $\mathrm{y}$, en general, de incapacidad) y confianza (confianza en la propia capacidad para resolver problemas; creencia en la propia independencia y capacidad de decidir por sí mismo). La adaptación española de esta prueba ha demostrado adecuadas propiedades psicométricas (González, Fernández, Pérez y Santamaría, 2004).

Personalidad. Para evaluar las dimensiones de personalidad de Neuroticismo y Extraversión utilizamos las subescalas correspondientes del Big Five-44 (BFI-44; Benet-Martínez y John, 1998), una prueba de autoinforme que ha mostrado adecuadas propiedades psicométricas y una alta correlación con medidas más extensas de los Cinco Grandes factores de personalidad. La adaptación al castellano de esta prueba ha mostrado características psicométricas similares a la versión original (Benet-Martínez y John, 1998).

\section{Procedimiento}

Este trabajo se incluye dentro de un proyecto de investigación más amplio acerca de la relación entre habilidades socio-emocionales y el ajuste psicosocial en la adolescencia. En este estudio las pruebas fueron completadas dentro del horario lectivo, principalmente en horario de tutorías, y en presencia del investigador, se garantizó en todo momento la voluntariedad y el anonimato de los participantes y se contó con la aprobación tanto de los diferentes centros como de los padres de los adolescentes.

\section{RESULTADOS}

\section{Análisis descriptivos y relaciones bivariadas}

En la tabla 1 se muestran los estadísticos descriptivos y los resultados del análisis de correlación de Pearson de las medidas utilizadas en el estudio. Como 
podemos observar, el nivel de percepción emocional se relacionó de forma significativa con todas las variables de ajuste psicosocial analizadas. De forma específica, aquellos adolescentes que mostraron una mayor habilidad a la hora de percibir emociones en los demás informaron de un mayor nivel de confianza en sí mismos, menor frecuencia de sentimientos de incapacidad y estrés social, y mejores relaciones tanto con sus iguales como con sus padres. Por otra parte, no se encontraron correlaciones significativas entre el nivel de percepción emocional y ninguna de las variables de personalidad evaluadas.

\begin{tabular}{|c|c|c|c|c|c|c|c|c|c|c|c|}
\hline & & 1 & 2 & 3 & 4 & 5 & 6 & 7 & 8 & Media & $D T$ \\
\hline $\begin{array}{l}\text { 1.Percepción } \\
\text { emocional }\end{array}$ & & -- & & & & & & & & 15.20 & 3.94 \\
\hline 2. Confianza & & $.20 * *$ & -- & & & & & & & 0.78 & 0.17 \\
\hline 3. Estrés social & & $-.20 * *$ & $-.30 * *$ & -- & & & & & & 0.22 & 0.21 \\
\hline $\begin{array}{l}\text { 4.Relaciones } \\
\text { interpersonales }\end{array}$ & & $.25^{* *}$ & $.45^{* *}$ & $-.62 * *$ & -- & & & & & 0.87 & 0.16 \\
\hline $\begin{array}{l}\text { 5.Relación con } \\
\text { padres }\end{array}$ & & $.18^{* *}$ & $.40 * *$ & $-.43^{* *}$ & $.39 * *$ & -- & & & & 0.71 & 0.19 \\
\hline $\begin{array}{l}\text { 6.Sentimientos } \\
\text { incapacidad }\end{array}$ & de & $-.26 * *$ & $-.34 * *$ & $.63 * *$ & $-.53^{* *}$ & $-.29 * *$ & -- & & & 0.26 & 0.20 \\
\hline 7.Extraversión & & .03 & $.19 * *$ & $-.18 * *$ & $.27 * *$ & .02 & $-.20 * *$ & -- & & 3.36 & 0.64 \\
\hline 8.Neuroticismo & & -.11 & $-.17 * *$ & $.24 * *$ & $-.24 * *$ & $-.16^{*}$ & $.27 * *$ & -.01 & -- & 4.8 & 0.56 \\
\hline
\end{tabular}

En cuanto a la relación entre la personalidad y el ajuste psicosocial, las dimensiones de neuroticismo y extraversión mostraron un patrón de correlaciones inverso. De este modo, mientras que un mayor neuroticismo se relacionó con una menor confianza de los adolescentes en sí mismos, peores relaciones interpersonales y con sus padres, y un mayor nivel de estrés en las situaciones sociales y sentimientos de incapacidad, la extraversión correlacionó de forma positiva con el nivel de confianza y relaciones interpersonales y de forma negativa con el estrés social y la incapacidad.

\section{Análisis de regresión}

Para comprobar si la percepción emocional predecía el ajuste psicosocial de los adolescentes una vez controlados los efectos de las variables socio-demográficas y las dimensiones de neuroticismo y extraversión, llevamos a cabo diferentes análisis de regresión jerárquica de forma separada para cada una de las variables criterio. En cada análisis, en un primer paso se introdujeron la edad y el sexo como covariables, en un segundo paso se introdujeron las dimensiones de neuroticismo y extraversión, y en el último paso se introdujeron las puntuaciones en percepción emocional. Los resultados aparecen en la tabla 2.

Como podemos observar, la percepción emocional se mostró como un predictor significativo de todas las variables psicosociales analizadas, incluso tras controlar el efecto de la edad, el sexo y las dimensiones de personalidad. En concreto, la 
percepción emocional apareció como un predictor positivo del nivel de confianza, relaciones interpersonales y relación con los padres, y como un predictor negativo del nivel de estrés social y de la aparición de sentimientos de incapacidad en los adolescentes. Los resultados también muestran la implicación de las variables de personalidad a la hora de predecir el ajuste psicosocial, así, mientras que el neuroticismo fue un predictor significativo de todas las variables analizadas (prediciendo de forma positiva el nivel de estrés social e incapacidad y de forma negativa el nivel de confianza y de relaciones con iguales y padres), la extraversión predijo significativamente el grado de confianza de los adolescentes y de bienestar con sus relaciones interpersonales (de forma positiva) así como el nivel de estrés social e incapacidad (de forma negativa). Por último, la edad apareció como un predictor positivo de los sentimientos de incapacidad (mostrando en mayor grado estos sentimientos los adolescentes de mayor edad) mientras que las mujeres informaron de mayor bienestar en sus relaciones sociales que los hombres.

Tabla 2. Análisis de regresión múltiple de la percepción emocional sobre las variables psicosociales

\begin{tabular}{|c|c|c|c|c|c|c|c|c|c|c|c|c|c|c|c|}
\hline & \multicolumn{3}{|c|}{ Confianza } & \multicolumn{3}{|c|}{ Estrés social } & \multicolumn{3}{|c|}{$\begin{array}{c}\text { Relaciones } \\
\text { interpersonales }\end{array}$} & \multicolumn{3}{|c|}{$\begin{array}{c}\text { Relación con los } \\
\text { padres }\end{array}$} & \multicolumn{3}{|c|}{$\begin{array}{c}\text { Sentimientos de } \\
\text { incapacidad }\end{array}$} \\
\hline & $R^{2}$ & $F$ & $\beta$ & $R^{2}$ & $F$ & $\beta$ & $R^{2}$ & $F$ & $\beta$ & $R^{2}$ & $F$ & $\beta$ & $R^{2}$ & $F$ & $\beta$ \\
\hline Paso 1 & .02 & 2.65 & & .003 & 0.39 & & .06 & 7.67 & & .002 & 0.02 & & .06 & 7.82 & \\
\hline Edad & & & -.06 & & & -.06 & & & -.05 & & & .01 & & & $.21^{*}$ \\
\hline Sexo & & & .07 & & & -.02 & & & $.18^{*}$ & & & -.01 & & & .06 \\
\hline Paso 2 & .07 & 4.70 & & .09 & 5.96 & & .16 & 11.66 & & .03 & 2.19 & & .15 & 10.53 & \\
\hline Extraversión & & & $.16^{*}$ & & & $-.16^{*}$ & & & $.22^{* *}$ & & & .01 & & & $-.18^{*}$ \\
\hline Neuroticismo & & & $-.14^{*}$ & & & $.23^{* *}$ & & & $-.21^{* *}$ & & & $-.17^{*}$ & & & $.21^{* *}$ \\
\hline Paso 3 & .10 & 5.34 & & .11 & 5.86 & & .19 & 11.46 & & .05 & 2.73 & & .19 & 11.20 & \\
\hline $\begin{array}{l}\text { Percepción } \\
\text { emocional }\end{array}$ & & & $.17^{*}$ & & & $-.14^{*}$ & & & $.18^{*}$ & & & $.14^{*}$ & & & $-.20^{*}$ \\
\hline
\end{tabular}

\section{DISCUSION}

Los resultados de nuestro estudio aportan evidencias sobre la importancia que las habilidades emocionales poseen a la hora de predecir el ajuste psicosocial de los adolescentes. De forma concreta, aquellos adolescentes que mostraron una mayor destreza a la hora de identificar el estado emocional de otras personas informaron igualmente de mejores relaciones sociales, una mejor consideración hacia sus padres y mayor percepción de sentirse estimados por éstos, y un menor nivel de sentimientos de estrés y tensión en las relaciones sociales. La percepción emocional predijo también de forma significativa el nivel de confianza y competencia percibida por los adolescentes. Además, estos efectos se mantuvieron incluso tras controlar la influencia del sexo, la edad, así como de importantes dimensiones de personalidad. 
Nuestros resultados están en consonancia con los encontrados por otros trabajos realizados en población adulta y que evalúan la IE a través de medidas de ejecución (Mayer et al., 2008), así como con otros estudios que, considerando la percepción emocional como una de las habilidades integradas dentro del conocimiento emocional, han demostrado su implicación a la hora de predecir la competencia social y académica en niños (Izard et al., 2001). Dentro del modelo de IE de Mayer y Salovey, la percepción emocional supone el primer paso en el procesamiento de la información emocional. Si las emociones no son adecuadamente percibidas, difícilmente las personas podrán hacer un uso adecuado de ellas. En este sentido, es posible que aquellos adolescentes con mejor habilidad a la hora de saber cómo se sienten las personas de su entorno utilicen dicha información no sólo para mostrar una mayor empatía hacia ellos, o para ayudarles a regular las emociones que sienten, sino también para modificar su comportamiento y adecuarse mejor a las situaciones sociales. Esto explicaría la relación positiva encontrada en nuestro estudio entre la percepción emocional y el bienestar con las relaciones interpersonales y con los padres. Además, en la medida en que la habilidad de percepción emocional ayude a los adolescentes a adaptarse a su entorno social, éstos acabarán mostrando menos sentimientos de estrés y tensión en su vida social, tal y como muestran nuestros resultados.

La literatura también sugiere que la capacidad para percibir con precisión las emociones de uno mismo está relacionada con la capacidad para evaluarlas en los demás (Zuckerman, Hall, DeFrank y Rosenthal, 1976). Así, la percepción emocional interpersonal se ha relacionado de forma general con un mayor conocimiento emocional y una mejor regulación de las emociones (Extremera, Fernández-Berrocal y Salovey, 2006; Izard et al., 2001). Quizás este sea uno de los mecanismos explicativos que subyacen a la relación entre percepción emocional y ajuste personal encontrada en nuestro estudio. Si las personas que reconocen adecuadamente las emociones ajenas son capaces de generalizar esta habilidad a su propia vida emocional, se beneficiarían de la información que sus sentimientos le aportan permitiendo un adecuado procesamiento de la misma. Otra explicación posible hace referencia al hecho de que la asociación entre percepción emocional y ajuste personal esté mediada por los propios beneficios sociales que esta habilidad supone. Desde esta perspectiva, la percepción emocional ejercería un efecto positivo sobre el ajuste social de los adolescentes algo que, a su vez, repercutiría en un mayor sentimiento de competencia y una mayor confianza por parte de éstos.

Nuestro estudio ha supuesto uno de los primeros trabajos llevados a cabo en población adolescente española en los que se analiza la influencia de la percepción emocional, como una de las habilidades integradas dentro del modelo de IE de Mayer y Salovey, sobre diferentes indicadores de ajuste personal y social. Futuras investigaciones deberán continuar esta línea de investigación corroborando los resultados aquí encontrados a través de diseños prospectivos que permitan inferir relaciones de causalidad entre las variables estudiadas y examinando la influencia de la percepción emocional sobre otras variables criterio importantes en la adolescencia, como la salud mental, la adaptación escolar o el nivel de rendimiento académico. Por otra parte, el avance en la investigación de la IE en la adolescencia necesita de un esfuerzo de los investigadores por diseñar medidas de ejecución que permitan evaluar todas las ramas 
integradas dentro de esta inteligencia y que demuestren su validez en la población adolescente.

Por último, es de resaltar que los resultados obtenidos aquí ofrecen apoyo empírico a los programas de intervención que, centrados en el entrenamiento de la IE, persiguen el objetivo de mejorar el bienestar psicosocial de los adolescentes y la convivencia en el aula. La habilidad de reconocer las emociones en los demás es susceptible de ser entrenada, desarrollada y mejorada. Desde esta perspectiva, la mejora de esta competencia puede ser un aspecto importante para aquellos programas dirigidos a fomentar las relaciones sociales positivas y/o a prevenir la aparición de problemas psicológicos y emocionales.

\section{REFERENCIAS}

Benet-Martínez, V. y John, O.P. (1998). Los Cinco Grandes across cultures and ethnic groups: Multitrait-multimethod analyses of the Big Five in Spanish and English. Journal of Personality and Social Psychology, 75, 729-750.

Brackett, M.A., y Mayer, J.D. (2003). Convergent, discriminant and incremental validity of competing measures of emotional intelligence. Personality and Social Psychology Bulletin, 29, 1147-1158.

Brackett, M.A., Rivers, S.E., Shiffman, S., Lerner, N. y Salovey, P. (2006). Relating emotional abilities to social functioning: a comparison of self-report and performance measures of emotional intelligence. Journal of Personality and Social Psychology, 91, 780-95

Brackett, M.A., Warner, R.M. y Bosco, J. (2005). Emotional intelligence and relationship quality among couples. Personal Relationships, 12, 197-212

Ciarrochi, J.V., Chan, A., Caputi, P. y Roberts, R. (2001). Measuring emotional intelligence (EI). En J.V. Ciarrochi, J.P. Forgas y J.D. Mayer (Eds.), Emotional intelligence in everyday life (pp. 25-44). Philadelphia, PA: Psychology Press.

Cosmides, L. y Tobby, J. (2000). Evolutionary psychology and the emotions. En M. Lewis y J.M. Haviland-Jones (Eds.), Handbook of emotions. New York: Guilford Press.

Damasio, A.R. (1994). El error de Descartes. Barcelona: Crítica.

Ekman, P. (2003). Emotions revealed. New York: Times Books, Henry Holt y Co.

Extremera, N. y Fernández-Berrocal, P. (2004). Inteligencia emocional, calidad de las relaciones interpersonales y empatía en estudiantes universitarios. Clínica y Salud, 15(2), 117-137.

Extremera, N., Fernández-Berrocal, P., Ruiz-Aranda, D. y Cabello, R. (2006). Inteligencia emocional, estilos de respuesta y depresión. Ansiedad y Estrés, 12, 191-205.

Extremera, N., Fernández-Berrocal, P. y Salovey, P. (2006). Spanish Version of the MayerSalovey-Caruso Emotional Intelligence Test (MSCEIT) Version 2.0: Reliabilities, Age, and Gender Differences. Psicothema, 18, 42-48.

Extremera, N., Salguero, J.M., Fernández-Berrocal, P. y Ruiz-Aranda, D. (2009). Acercamientos actuales en la medición científica de la inteligencia emocional: algunas implicaciones de su uso en el ámbito laboral. En J.M. Augusto (Ed.), Estudios en el ámbito de la inteligencia emocional (pp. 175-198). Jaén: Huarte de San Juan, Psicología.

Fridja, N.H. (1988). The laws of emotion. American Psychologist, 43, 349-358.

González, J., Fernández, S., Pérez, E. y Santamaría, P. (2004). Spanish adaptation of Behavior Assessment System for Children and Adolescentes: BASC. Madrid: TEA Ediciones.

Izard, C.E. (2001). Emotional intelligence or adaptive emotions? Emotion, 1(3), 249-257. 
Izard, C.E., Fine, S.E., Schultz, D., Mostow, A.J., Ackerman, B.P. y Youngstrom, E.A. (2001). Emotion knowledge as a predictor of social behavior and academic competence in children at risk. Psychological Science, 12, 18-23.

Katelaar, T. y Todd, P.M. (2001). Framing our thoughts: Ecological rationality as evolutionary psychologist's answer the frame problem. En H.R. Holcomb III (Ed.), Conceptual challenges in evolutionary psychology. Innovative research strategies. London: Kluwer.

Keltner, D. y Haidt, J. (2001). Social functions of emotions. En T.J. Mayne y G.A. Bonanno (Eds.), Emotions: Currents issues and future directions. Emotions and social behavior (pp. 192-213). New York: Guilford.

Lopes, P.N., Brackett, M.A., Nezlek, J.B., Schutz, A., Sellin, I. y Salovey, P. (2004). Emotional intelligence and social interaction. Personal Social Psychological Bulletin, 30(8), 10181034.

Lopes, P.N., Salovey, P., Cote, S. y Beers, M. (2005). Emotion regulation abilities and the quality of social interaction. Emotion, 5(1), 113-118.

Martorell, C., González, R., Rasal, P. y Estellés, R. (2009). Convivencia e inteligencia emocional en niños en edad escolar. European Journal of Education and Psychology, 2(1), 69-78.

Mayer, J.D., Roberts, R.D. y Barsade, S.G. (2008). Human Abilities: Emotional Intelligence. Annual Review of Psychology, 59, 507-536.

Mayer, J.D. y Salovey, P. (1997). What is emotional intelligence? En P. Salovey y D.J. Sluyter (Eds.), Emotional development and emotional intelligence: Implications for educators (pp. 3-31). New York: Basic Books.

Mayer, J.D., Salovey, P. y Caruso, D. (2000). Models of emotional intelligence. In R.J. Sternberg (Ed.), Handbook of intelligence (pp. 396-420). New York: Cambridge.

Mayer, J.D., Salovey, P. y Caruso, D. (2001). Technical Manual for the MSCEIT v. 2.0. Toronto, Canada: MHS Publishers.

Nesse, R.M. (1990). Evolutionary explanations of emotions. Human Nature, 1, 261-289.

Overskeid, G. (2000). The slave of passions: Experiencing problems and selecting solutions. Review of General Psychology, 4, 483-522.

Plutchik, R. (1984). Emotions: A general psihoevolutionary theory. En K. R. Scherer y P. Ekman (Eds.), Approaches to emotion (pp. 197-219). Hillsdale, NJ: Erlbaum.

Ramos, N., Fernández-Berrocal, P. y Extremera, N. (2007). Perceived Emotional intelligence facilitates Cognitive-Emotional Processes of Adaptation to an Acute Stressor. Cognition y Emotion, 21, 758-772.

Reynolds, C.R. y Kamphaus, R.W. (2004). Behavior assessment system for children. Circle Pines, $\mathrm{MN}$ : American Guidance Service.

Salovey, P. y Mayer, J.D. (1990). Emotional intelligence. Imagination, Cognition \& Personality, 9, 185-211.

Salovey, P., Mayer, J.D., Goldman, S.L., Turvey, C. y Palfai, T.P. (1995). Emotional attention, clarity, and repair: Exploring emotional intelligence using the trait meta-mood scale. En J.W. Pennebaker (Ed.), Emotion, disclosure \& health (pp. 125-151). Washington: American Psychological Association.

Zeidner, M., Matthews, G. y Roberts, R. (2008).The science of emotional intelligence: current consensus and controversies. European Psychologist, 13(1), 64-78.

Zuckerman, M., Hall, J.A., DeFrank, R.S. y Rosenthal, R. (1976). Encoding and decoding of spontaneous and posed facial expressions. Journal of Personality and Social Psychology, 34, 966-977. 